\title{
Correlation analysis between the Aerosol Optical Depth and Cosmic Rays
}

\author{
Maghrabi A. $\mathbf{H}^{\mathbf{1}}$ \\ National Centre for Applied Physics, King Abdulaziz City For Science and Technology \\ P.O.Box: 6086; Riyadh 11442, Saudi Arabia.
}

\section{Kudela K}

Institute of Experimental Physics

Slovak Academy of Sciences, Košice, Slovakia

kkudela@saske.sk

In this study, aerosol optical depth (AOD870 nm) data, for the period between 1999 to 2015 obtained from the solar village AERONET site (central Saudi Arabia); have been correlated with cosmic ray (CR) data from Oulu neutron monitor. Positive and significant correlation between the two variables has been found. Moreover, power spectral analyses using the Fourier Transform (FT) technique have been carried out to investigate the periodicities in both the CR and AOD time series. Several common periodicities between the two variables have been recognized indicating possible mutual relation between variations of aerosols and of CR intensity observed on the ground.

amaghrabiekacst.edu.sa

36th International Cosmic Ray Conference -ICRC2019-

July 24th - August 1st, 2019

Madison, WI, U.S.A.

\footnotetext{
${ }^{1}$ Maghrabi A

(c) Copyright owned by the author(s) under the terms of the Creative Commons 


\section{Introduction}

Atmospheric aerosols are considered as an important parameter of the Earth's atmospheric system. Absorption and scattering are the two main physical processes that are associated with the interaction of aerosol particles with both solar and long-wave radiation [e.g., 1-2]. They, also, affect cloud properties and composition processes, which therefore impact the Earth's radiative budget [3-4].

Aerosol Optical Depth (AOD) measures the extinction of a monochromatic light beam through a given aerosol layer and considered as one of most important parameters in characterizing the aerosols radiative forcing at the surface and at the top of the atmosphere[2,5].

Primary cosmic rays are high-energy particles persistently bombarding the Earth's atmosphere. Due to solar and heliospheric disturbances, primary cosmic rays are modulated on different time scales. This modulation leads to a change in atmospheric properties, which may influence the global weather and climate [e.g. 6]. Understanding this modulation has become an important subject that attracted scientists from different disciplines [3, 7].

At the top of the atmosphere primary CR particles interact with the atmospheric molecules and produce cascades of secondary particles. Because of their energies and their capacities to cause ionization at various atmospheric levels, CR particles can affect the atmospheric properties which influence its physical and chemical characteristics [e.g., 3].

Over the most recent two decades, a few researches have been carried out to examine the relationship between CRs and cloud cover [8-9]. In any case, the cloud cover may not be a dependable proportion of the cloud microphysical characteristics [10]. So as to explore the relationship between CRs and atmospheric properties, an alternate methodology is required.

In this study, correlation and Fourier transform investigations have been carried out to investigate the relationship between the CRs and aerosol optical depth. Cosmic ray data from Oulu neutron monitor and relating AOD and aerosol size distribution from solar village AERONET station have been used with the end goal of this work.

\section{Experimental Data}

Hourly CR data from Oulu neutron monitor $\left(\mathrm{R}_{\mathrm{c}} \sim 0.2 \mathrm{GV}\right)$ for the period between 19992015 were used. Additionally, hourly-averaged Level 1.5 products of the AOD data (at $870 \mathrm{~nm}$ ) for the same period were obtained from AERONET solar village station of Saudi Arabia were utilized [e.g., 5]. The day by day data of the AOD and CR data were averaged to get their monthly mean values. The least square method and related statistical tests were used in the first part of this study to examine the relationship between the AOD and the CR data. In addition, Fourier Transform analyses were utilized, afterward, to identify the Aerosol and cosmic ray quasi-periodicities in various frequency ranges. 


\section{Results and Discussions}

\subsection{Correlation results}

The inter-annual variations of the AOD870 $\mathrm{nm}$ and CR data for the period between 19992015 are depicted in figure 1 . The one year running mean is additionally presented. It can be seen that, both variables increase from 2003 and reached the maximum by 2009 (the minimum of the solar cycle 23).
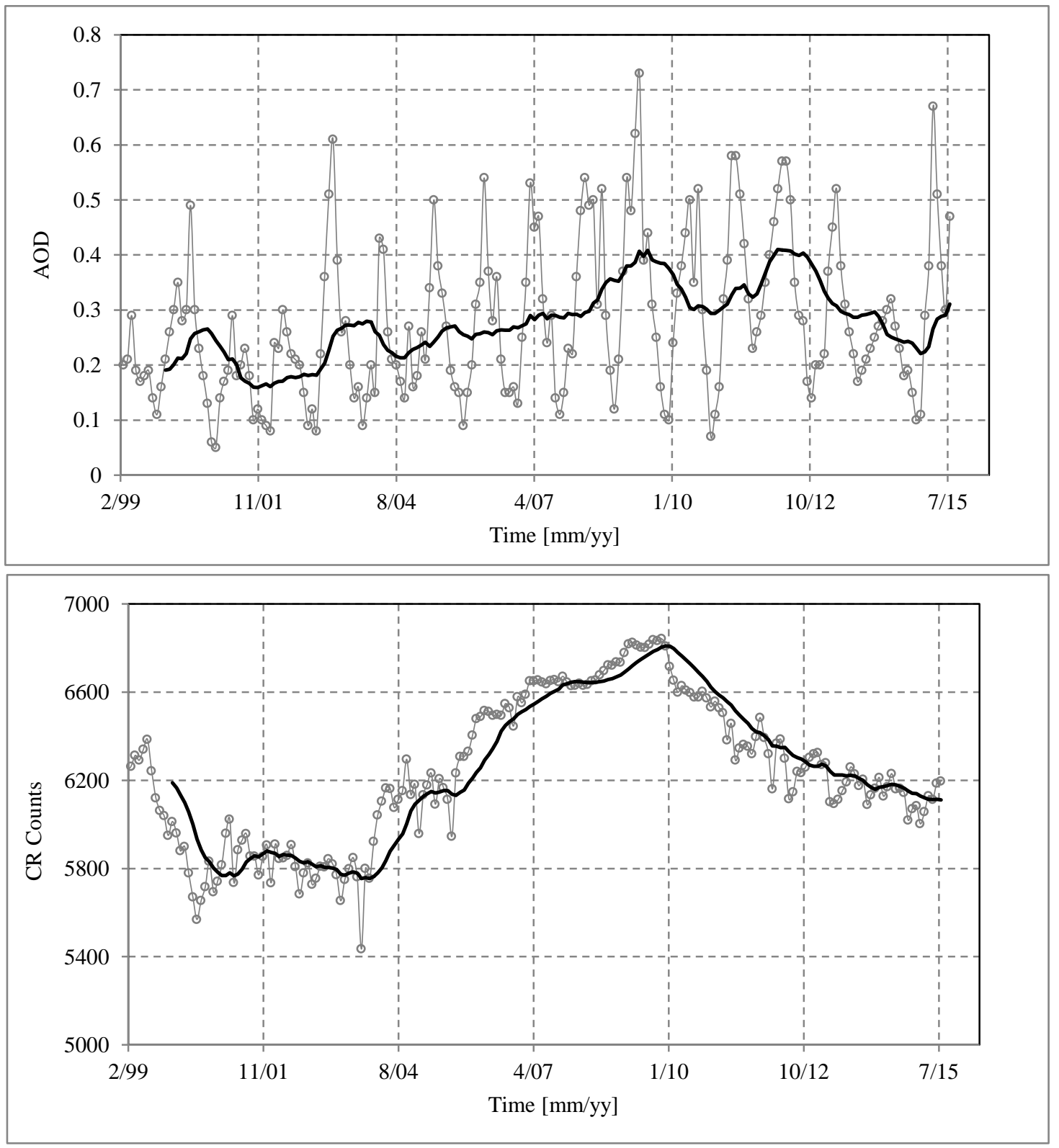

Fig. 1: monthly mean values of the AOD at $870 \mathrm{~nm}$ (top panel) and CR (bottom panel) data for the period between 1999-2015.The dark line is a year running mean line. 
The relationship between the mean monthly values of both the AOD8700 $\mathrm{nm}$ and the CR for the considered periods is indicated in fig.2. While there is spread in the data, the figure shows that the CRs are correlated with AOD870 $\mathrm{nm}$.

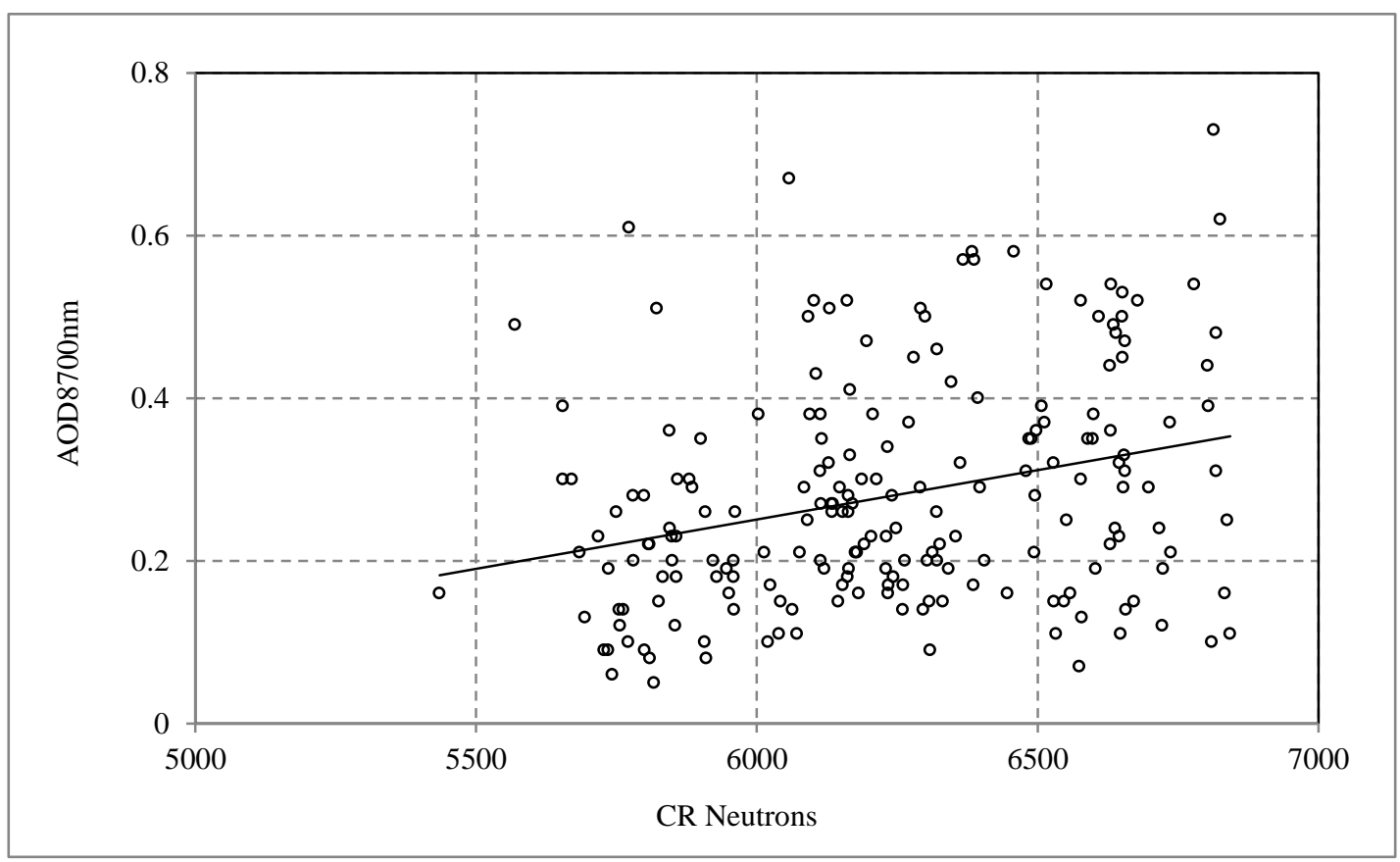

Fig. 2: scatter plots between monthly mean values of the CR neutrons and AOD at $870 \mathrm{~nm}$ during the study period. The solid line is the regression line.

The regression equation between the CR neutrons and AOD870 $\mathrm{nm}$ was:

$$
A O D_{870}=0.00012 C_{\text {Neutron }}-0.47
$$

The equation demonstrates that AOD870 nm increases as the cosmic ray increases and vice versa. Despite the fact that the correlation coefficient of this equation was low (about 0.26), the relationship between the two variables is statistically significant, $\left(\mathrm{F}_{\text {value }}=17.8>>\right.$ the critical value $=6.90$ at $99 \%$ confidence level). Nevertheless, the correlations between cosmic rays and the atmospheric aerosols emerge from the fact that the cosmic ray particles are one of the main sources of atmospheric ionization [3-4, 7-9]. The high disperse in the relationship between the cosmic ray and the AOD870 $\mathrm{nm}$ values might be because of a few factors. These incorporate the presence of various aerosols types which originated from various sources [e.g., 11-12]. The sporadic solar events, for example, coronal mass ejections, may influence the CR intensities which may affect the above correlations. 


\subsection{Quasi-periodicities between the CR and AOD}

The power spectrum density of time series of the AOD870 nm and the CR count rates is analyzed and presented in Fig.3.

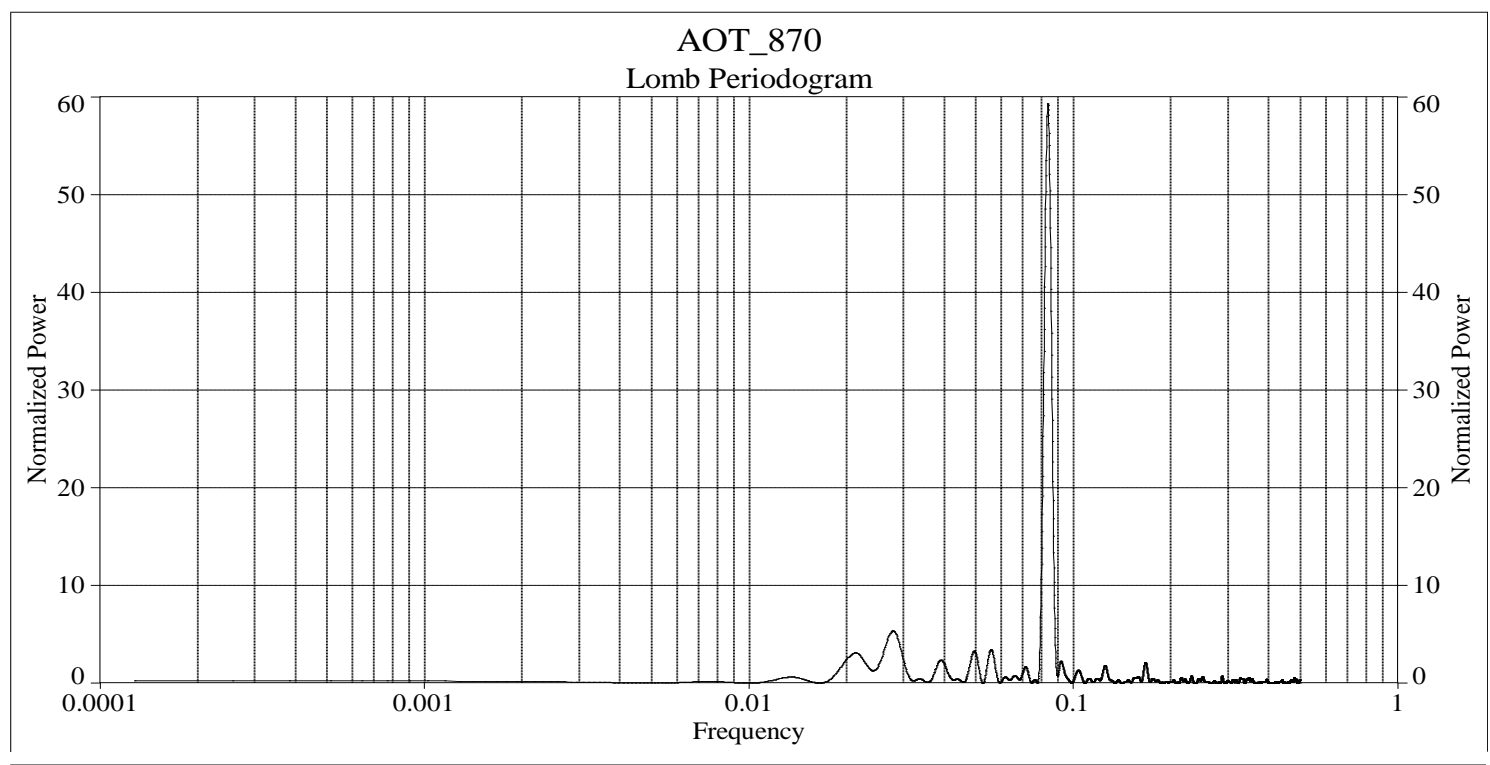

CR Data

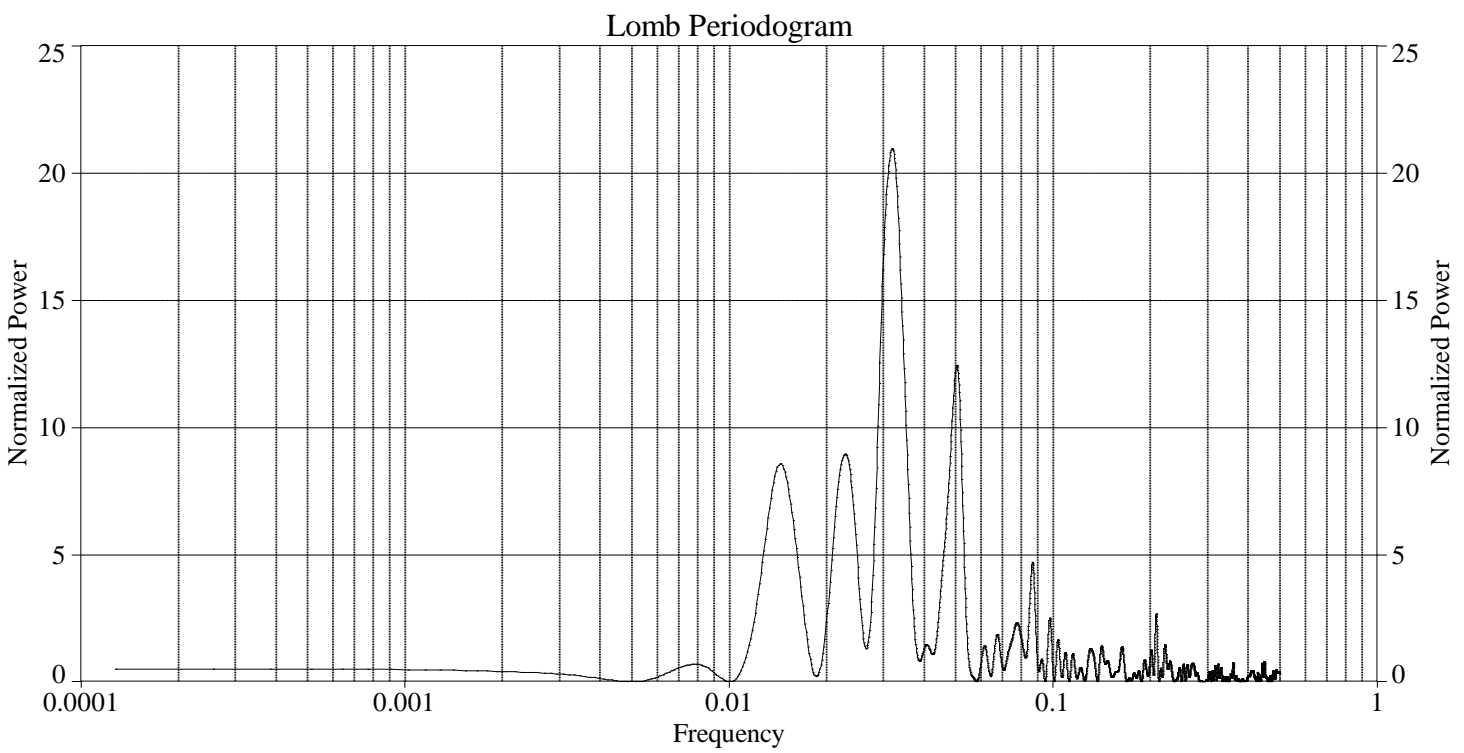

Fig. 3: Power spectral density (PSD) of monthly time series of AOD $870 \mathrm{~nm}$ (top panel) and CRs (bottom panel), for timespan 1999/2 - 2015/7. PSD figured by FFT strategy. 
The two spectra differ slightly in their shapes and the strength of the peaks. However, several common periods with various strength and positions are found in the low frequencies. For the interval somewhere in the range of 0.02 and 0.5 , the CR has significant peaks at 5.8 years, 3.6 years, 2.6 years, and 1.66 years. On the other hand the AOD spectrum shows strong seasonal ( 1 year) component not plainly found in CR. This is mainly due to the atmospheric source. Other periodicities, such as the 3.6 years, 2.1 years, and 1.7 years are presented in the AOD data. It is clearly, evident that the 3.6 years and 1.7 years are common Quasi-periodicities between the CR and AOD time series. These periodicities are similar to those reported by several investigators and found in solar, interplanetary, and cosmic ray parameters [1318]. The results presented in this study are preliminary, more detailed investigations of time series of atmospheric aerosol measurements and CR data at various locations and for longer time periods are recommended.

\section{Conclusions:}

The aerosols optical depth (AOD $870 \mathrm{~nm}$ ) measurements obtained from AERONET site located in central Saudi Arabia and the cosmic ray data acquired from Oulu neutron monitor for the period between 1999 - 2015 were utilized to study their relationships.

It was found that there is a significant, positive, correlation between the time series of CR with time series of aerosols. This can be explained by the fact that the cosmic ray particles are considered as the fundamental sources of atmospheric ionization which prompts a composition of atmospheric aerosols. Power spectral investigation for the considered data gives an indication of the presence of 3.6 and $~ 1.7$-year quasi-periodicity. The correlation and power spectral examinations show the conceivable common relation between variations of aerosols and CR intensity observed on the ground at explicit position investigated here.

\section{Acknowledgments}

The authors would like to thank King Abdulaziz City for Science and Technology (KACST) for supporting this work. 


\section{References}

[1] J. Haywood and O. Boucher. 2000. Estimates of the direct and indirect radiative forcing due to tropospheric aerosols. A review. Rev. Geophys.38, 513-543.

[2] A. Maghrabi and R. Alotaib R 2018. Long-term variations of AOD from an AERONET station in the central Arabian Peninsula, 2017; Theoretical and Applied Climatology, 134,(3-4),

[3] J. Kristjansson et al., 2002. A new look at possible connections between solar activity, clouds and climate. Geophys. Res. Lett. 29, 10.1029/2002GL015646.

[4] A. Rawal et al., 2013. Quantifying the importance of galactic cosmic rays in cloud microphysical processes. J. Atmos. Sol.-Terr. Phys.102.

[5] B. Holben et al., 2001. An emerging ground-based aerosol climatology: aerosol optical depth from AERONET. J. Geophys. Res.106.

[6] Haigh, J. 1996. The impact of solar variability on climate. Science 272, 981-984.

[7] D. Singh and R. Singh. 2010. The role of cosmic rays in the Earth's atmospheric processes Pramana - J. Phys. 74, 153

[8] H. Svensmark, et al., 2009. Cosmic ray decreases affect atmospheric aerosols and clouds. G. Res. Lett. 36, L15101.

[9] H. Svensmark H, et al., .2017. Increased ionization supports growth of aerosols into cloud condensation nuclei. Nat. Commun. 8(1), 2199.

[10] T. Sloan, and A. Wolfendale, 2008. Testing the proposed causal link between cosmic rays and cloud cover, Environ. Res. Lett. 3024001.

[11] H. Kutiel, and H. Furman, 2003. Dust storms in the Middle East: Sources of origin and their temporal characteristics. Indoor and Built Environment 12(6), 419-426.

[12] A. Maghrabi and A. Al-Dosari. 2016. Effects on surface meteorological parameters and radiation levels of a heavy dust storm occurred in Central Arabian Peninsula. Atmos. Res. 182, 30-35.

[13] P. Chowdhury and K. Kudela, 2018. Quasi-periodicities in cosmic rays and time lag with the solar activity at a middle latitude neutron monitor: 1982-2017. Astrophys. Space Sci. 363: 250, 17.

[14] M. El Borie and S. Al Thoyaib, 2002. Power spectrum of cosmic-ray fluctuations during consecutive solar minimum and maximum periods. Solar Phys. 209(2).

[15] H. Mavromichalaki H, et al., 2003. Low and high frequency spectral behavior of cosmic ray intensity for the period 1953-1996. Ann. Geophys, 21:1681-9.

[16] J. Valdes-Galicia, J., et al., 1996. The cosmic-ray 1.68-year variation: A clue to understand the nature of the solar-cycle. Sol.Phys. 167.

[17] J. Pap, J., et al., 1990. Periodicities of solar irradiance and solar activity indices, Solar Phys., 129.

[18] A. Antalova, A., et al., 2000. The solar and cosmic-ray synodic periodicity (1969-1998), ESA SP463, Space Sci. Rev. 97. 